\title{
On the use of Convex Optimization for Array Synthesis Problems
}

\author{
Benjamin Fuchs \\ Institute of Electronics and Telecommunications of Rennes, UMR CNRS 6164, University of Rennes 1, France \\ e-mail: Benjamin.fuchs@univ-rennes1.fr
}

The synthesis of antenna arrays is a very long standing field in electromagnetism because of its many applications (e.g. radar, radio astronomy, sonar, communications, direction-finding, seismology, medical diagnosis and treatment). A host of methods have been proposed since the 40 's to solve increasingly difficult array synthesis problems. These techniques range from analytical methods (fast but limited to very specific problems) to global optimization approaches (comprehensive but limited in performances due to their computational burden). Convex optimization has been shown to be a good trade-off in efficiency/generality between analytical and global optimization techniques in a number of relevant cases.

A variety of antenna synthesis problems can be cast as convex optimization ones. Such a convex formulation has been proposed for the synthesis of focused beams [1], sparse arrays [2,3], polarization [4] and arrays with excitation control [5] for instance. The objectives of these synthesis problems are respectively: the minimization of the side lobe level, the minimization of the number of antennas, the optimization of the radiated wave polarization and the regularization of the antenna excitations whereas the constraints of these synthesis problems are typically a radiation pattern template.

A number of frequently encountered array synthesis problems are inherently non convex such as the synthesis of shaped beams, phase-only excitations or reconfigurable arrays. These problems can be approximated as convex optimization ones using the semidefinite relaxation technique in order to approximate the quadratic constraints arising in many array synthesis problems [6,7]. The power radiated by an antenna array of steering vector a and excitations $\mathbf{x}$ can be written:

$$
|f(\mathbf{x})|^{2}=\mathbf{x}^{H} \mathbf{A} \mathbf{x}=\operatorname{Trace}\left(\mathbf{A x} \mathbf{x}^{H}\right)
$$

where $\mathbf{A}=\mathbf{a a}^{H}$ is an Hermitian matrix, $\mathbf{a}^{H}$ standing for the conjugate transpose of $\mathbf{a}$. By introducing the variable $\mathbf{X}=\mathbf{x x}^{H}$, the radiated power becomes:

$$
|f(\mathbf{X})|^{2}=\operatorname{Trace}(\mathbf{A X}) \text { with } \mathbf{X} \geqslant 0 \text { and } \operatorname{rank}(\mathbf{X})=1
$$

where the only non convex rank constraint can be dropped, hence the relaxation. The price to pay for turning the initially difficult array synthesis problem of unknown $\mathbf{x}$ into an easier to solve convex one of unknown $\mathbf{X}$ is the increase in dimensions since the vector problem of dimension $N$ is transformed into a matrix one of size $N \mathrm{x} N$. Finally, there are many readily available solvers to solve convex optimization problems. For instance, CVX [8] can be used to find efficiently the optimal solution of such problems. Various representative examples of array synthesis problems solved via convex optimizations will be shown.

1 H. Lebret and S. Boyd, "Antenna pattern synthesis via convex optimization," IEEE Trans. Signal Processing, vol. 45, no. 3, pp. 526-531, Mar. 1997.

2. B. Fuchs, "Synthesis of sparse arrays with focused or shaped beampattern via sequential convex optimizations," IEEE Trans. on Antennas and Propag., vol. 60,no. 7, pp. 3499-3503, July 2012.

3. G. Prisco and M. D'Urso, "Maximally sparse arrays via sequential convex optimizations," IEEE Antennas Wireless Propag. Lett., vol. 11, pp. 192-195, Feb. 2012

4. B. Fuchs and J.J. Fuchs, "Optimal polarization synthesis of arbitrary arrays with focused power pattern," IEEE Trans. on Antennas and Propag., vol. 59, no. 12, pp. 4512-4519, Dec. 2011.

5. B. Fuchs and S. Rondineau, "Array Pattern Synthesis with Excitation Control via Norm Minimization," IEEE Trans. on Antennas and Propag., vol. 64, no. 10, pp. 4228-4234, Oct. 2016.

6. P. J. Kajenski, "Phase only antenna pattern notching via a semidefinite programming relaxation," IEEE Trans. Antennas Propag., vol. 60, no. 5, pp. 2562-2565, May 2012.

7. B. Fuchs, "Application of Convex Relaxation to Array Synthesis Problems," IEEE Trans. on Antennas and Propag., vol. 62, no. 2, pp. 634-640, Feb. 2014.

8. CVX Research, Inc. CVX : Matlab software for disciplined convex programming. Available from http ://cvxr.com/cvx. 